\title{
La missione del sacerdote nel pensiero del beato don Jerzy Popiełuszko - un martire contemporaneo della Polonia
}

\section{The Mission of the Priest in the Thought of Blessed Jerzy Popiełuszko - a Modern Priest-Martyr from Poland}

\begin{abstract}
The study entitled: La missione del sacerdote nel pensiero del beato don Jerzy Popietuszko - un martire contemporaneo della Polonia ("The Mission of the Priest in the thought of Bl. Jerzy Popiełuszko, a modern priest-martyr from Poland") explores the theology of priesthood in the light of the thought and example of Bl. Father Jerzy Popiełuszko. The essay covers the following issues: 1) Fr Jerzy Popiełuszko in the context of the times he lived in; 2) The mission of the priest is to be close to God and to people; 3) The mission of the priest is to proclaim the Good News; 4) The mission of the priest is to minister the Sacraments; 5) The mission of the priest is to nurture hope; 6) The mission of the priest is to die for the Faith. The Author concludes that the phenomenon of Fr Jerzy Popiełuszko, which came into its full force after his martyr's death, allows us to see him as a clear model for modern priests. The blessed martyr from Warsaw reminds us that sanctity can be attained and lived in all historical contexts. Fr Jerzy also comes across as an exemplar of a very engaged shepherd of souls who walked the path to holiness whilst also working closely with many lay people who showed a special readiness to work with him and solicitude for the good of the Church in a world at war against God, the Church and its priests.
\end{abstract}

\section{Keywords}

Bl. Jerzy Popiełuszko, priesthood, martyrdom, pastoral care, nurturing hope.

"Il Signore benedica la Polonia e doni sacerdoti con lo spirito evangelico di Popiełuszko, 25 maggio 2002" - ha scritto il card. Joseph Ratzinger nell'apposito Libro di memorie in occasione della visita nella parrocchia di san Stanislao Kostka a Varsavia, dove si trova la tomba del beato don Jerzy Popiełuszko, martire contemporaneo Polacco. 


\section{Introduzione}

"Il lavoro di un prete - scrisse don Jerzy (Giorgio) Popiełuszko - è la continuazione dell'opera di Gesù Cristo. Il prete è scelto tra la gente per la gente, per servirla. Perciò uno dei doveri del prete è stare lì, dove la gente è più bisognosa, ferita, maltrattata nella sua dignità" . Don Jerzy Popiełuszko non scrisse nessun trattato sulla missione del sacerdote e sui suoi impegni, e non scrisse neppure nessun manuale di teologia pastorale. Lavorando, invece, come sacerdote e sbrigando le faccende quotidiane mostrò un esempio della vita sacerdotale. Già due giorni dopo il suo funerale, nelle mani del primate di Polonia il card. Józef Glemp, gli operatori della sanità avevano presentato la prima richiesta di iniziare il processo di beatificazione, firmato da 178 persone. Un mese dopo la morte di don Jerzy Popiełuszko, la stessa richiesta venne avanzata dal vescovo Zbigniew Kraszewski insieme a dodici sacerdoti.

Il fenomeno della vita e del servizio di don Jerzy Popiełuszko, la sua eroica prontezza a dare la vita per Gesù Cristo e il suo coronamento nel martirio per la fede, ci inducono a fare una domanda su come lui interpretava la missione del sacerdote. Non è senza importanza anche il fatto che il suo messaggio penetrò tutta la Chiesa in Polonia e anche in tanti altri posti nel mondo. Nel presente articolo saranno espresse in dettaglio le questioni, che sembrano chiarire e caratterizzare il servizio sacerdotale di don Jerzy Popiełuszko. L'esempio della sua vita e del suo insegnamento lasciano distinguere cinque incarichi fondamentali del sacerdote. La sua missione comprende soprattutto: stare vicino a Dio, alla gente, impartire i sacramenti, confermare nella speranza e formare in sé la prontezza a morire per la fede.

\section{Il Beato Jerzy Popiełuszko sullo sfondo dei suoi tempi}

Don Jerzy Popiełuszko nacque il 14 settembre 1947 in Polonia, in un piccolo villaggio di nome Okopy. Nel secondo dopoguerra, alla Polonia fu imposto il governo comunista e si trovò in una forte dipendenza dall'ex U.R.S.S. Il giovane Popiełuszko nel 1965 entrò nel Seminario di San Giovanni Battista

1 J. Popiełuszko, ,, Jestem z tymi ludźmi w każdej sytuacji”, in: G. Bartoszewski, Zapiski. Listy i wywiady ks. Jerzego Popietuszki 1967-1984, Warszawa 2009, p. 114. 
a Varsavia. Erano i tempi in cui la Chiesa in Polonia veniva sistematicamente perseguitata. Già nel 1945 i comunisti ruppero unilateralmente il concordato con la Santa Sede e negli anni successivi cominciarono gli arresti, i processi pubblici dei preti, chiamati dalla propaganda comunista "banditi in abito talare" o "propagatori dell'ideologia straniera". Nel 1953 le autorità comuniste polacche, dopo consultazioni con le autorità sovietiche, arrestarono e isolarono per più di tre anni il Primate card. Stefan Wyszyński. Le repressioni riguardarono anche i seminaristi. Dopo il primo anno di studi Popiełuszko, così come tanti altri aspiranti sacerdoti, fu arruolato nell'esercito, in un'unità, creata proprio per i seminaristi, con una disciplina ferrea. Lì sperimentò un indottrinamento politico, persecuzioni e torture, che avevano come scopo di fargli passare la voglia di diventare prete e incoraggiarlo a collaborare con le autorità comuniste. Nonostante tutte queste esperienze e la salute cagionevole, nel 1968 tornò in seminario e dopo averlo concluso il 28 maggio 1972 ricevette l'ordinazione sacerdotale dal card. Stefan Wyszyński. Dopo questa data, svolse il suo servizio come vicario parrocchiale, cappellano degli studenti dell'Università e cappellano dei lavoratori della sanità. Nel $1980 \mathrm{fu}$ assegnato alla parrocchia di San Stanislao Kostka a Varsavia, in cui lavorò fino alla fine della sua vita - fino alla sua morte da martire, il 19 ottobre 1984. Proprio quel giorno don Jerzy Popiełuszko fu rapito dai funzionari dei Servizi di Sicurezza dello Stato comunista, brutalmente torturato, dopodiché legato, e con un sacco pieno di pietre attaccato al corpo, fu gettato nel fiume Vistola. Questo fu il modo attraverso il quale il regime comunista fece tacere per sempre don Jerzy, mostrando così il suo vero volto disumano. È significativo che nelle sue ultime parole rivolte ai fedeli, qualche ora prima di morire, disse: "Preghiamo per essere liberati dalla paura, dall'intimidazione e soprattutto dal desiderio di vendetta e violenza"

Oggi possiamo affermare che durante gli ultimi anni di vita, don Jerzy Popiełuszko giunse a una tale maturazione di fede e di unione con Cristo che lo rese pronto al martirio, tanto da poter dire: "Sono sicuro che tutto quello che

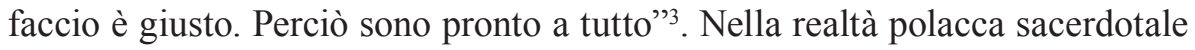
di allora, nel sistema totalitario, quelle parole significavano essere pronto

2 J. Popiełuszko, Rozważania różańcowe, in: M. Kindziuk, Ksiądz Jerzy zwyciężat dobrem, Warszawa 2009, p. 198.

3 J. Popiełuszko, ,, Jestem gotowy na wszystko”, in: G. Bartoszewski, Zapiski. Listy i wywiady ks. Jerzego Popietuszki 1967-1984, op. cit., p. 112. 
a donare la vita. In questi anni, inoltre, si incominciarono ad intravvedere dei piccoli segnali di cambiamento all'interno del sistemo politico totalitario, che portarono una ventata di speranza per il popolo polacco.

In gran parte quella speranza fu legata alla formazione del movimento sociale "Solidarność". Le aspettative del Popolo Polacco per la libertà e l'indipendenza dall'ex U.R.S.S. stavano diventando reali. Ma la risposta delle autorità comuniste della Repubblica Popolare Polacca fu la dichiarazione della legge marziale, il 13 dicembre 1981, che mirava a soffocare il movimento di rinnovamento sociale e morale della nazione. Ciò significava l'inizio di persecuzioni per le persone legate a "Solidarność". Questa situazione portò don Jerzy Popiełuszko a intensificare ancor di più la sua preghiera e a dedicare una maggiore cura alla vita spirituale di tutte le persone ferite e sofferenti, provvedendo anche al loro aiuto materiale.

\section{La missione del sacerdote è stare vicino a Dio e alle persone}

Raccontando la sua esperienza intima con Dio e descrivendo la gioia di sentire la sua presenza nella sua vita quotidiana, don Jerzy scrisse nei suoi appunti: "Oggi, prima di pranzo, alle 12.10 sono andato a recitare il rosario nel coro della chiesa. Silenzio benedetto. Sopra il pulpito una croce d'oro. Di tanto in tanto appariva un raggio di sole e la croce si rischiarava di una calda luminosità tenue e dorata. Dopo veniva l'oscurità. Dio, com'è simile alla vita umana, grigia, difficile e a volte cupa, che sarebbe insopportabile se non ci fossero i raggi della Tua presenza, il segno che sei con noi, sempre lo stesso, buono e pieno di amore". Vivere alla presenza di Dio, la ricerca dei semplici segni della bontà e dell'amore del Signore, portavano don Jerzy verso le persone, per stare con loro così come Dio ovunque sta con il suo popolo: nella felicità e nella sfortuna, nella gioia e nella sofferenza. Così anche quel sacerdote voleva stare con loro per avvicinarli a Dio con la sua presenza. Nulla di strano se con fermezza e zelo, tipici della cura per la salvezza delle anime, scriveva: "Non so più chiudere il mio servizio sacerdotale in chiesa, anche se così tanti «consiglieri» mi dicono che un

4 J. Popiełuszko, Zeszyt zielony, 25 XII [1983], in: G. Bartoszewski, Zapiski. Listy i wywiady ks. Jerzego Popiełuszki 1967-1984, op. cit., p. 80. 
vero prete polacco non ne dovrebbe mai uscire. Starò tra i miei operai, finché mi sarà possibile..."

Secondo lui, i sacerdoti - gli uomini di Chiesa - dovrebbe cercare di stare anche tra coloro che non credono, che dubitano. Dovrebbero fare compagnia all'uomo, specialmente quando soffre e vive delle esperienze difficili. In un'intervista ricorda che grazie alla scoperta dell'atteggiamento disponibile degli uomini di Chiesa a beneficio dell'uomo - atteggiamento che nasce dalla fede - molti dubbiosi e i lontani spesso hanno creduto e hanno cominciato ad accostarsi ai sacramenti ${ }^{6}$.

La missione del sacerdote è anche presenza tra la gente. Secondo don Jerzy stare con le persone appare come il suo compito fondamentale: essere a disposizione, dedicare il proprio tempo, ascoltare. In un'intervista spiega in che modo capisce la presenza del prete tra la gente: "La parte più importante è l'aiuto spirituale del prete. Che cosa posso dare? Soprattutto il mio tempo e la mia preghiera. Semplicemente sto con loro in ogni situazione. La gente viene, sa che casa mia è aperta per loro dalla mattina alla sera. Ogni giorno vengono tante persone che non necessariamente si aspettano un aiuto di carattere materiale. Vogliono dire qualcosa, piangere il loro dolore, parlare con gli altri dei loro problemi. Quando li capisco, quando a volte ascoltandoli ho le lacrime agli occhi, mettendomi al loro posto e immedesimandomi nei loro problemi, questo molto spesso rappresenta molto più di un aiuto materiale"7. Dalle testimonianze dei laici che collaboravano con don Jerzy e che erano impegnate nelle sue attività, risulta che aveva una particolare abilità nell'ascoltare la gente in modo attento. Per loro non era tanto un consigliere ma piuttosto un confidente. Tante persone andavano da lui per stare con lui, per parlargli delle loro sofferenze, delle loro miserie e paure. Lo stesso don Popiełuszko parlava del suo lavoro: "La gente, dopo molti anni, spesso più di dieci, acquista il coraggio di venire da me a chiedere la riconciliazione con Dio, la confessione e la comunione. È un'emozione forte per me in quanto prete, come anche per queste persone. Non hanno osato andare da nessun altro. Molto spesso il processo di conversione, del ritorno a Dio, alla Chiesa o in generale la scoperta

5 J. Popiełuszko, ,Osiadtem” wśród robotników, in: G. Bartoszewski, Zapiski. Listyi wywiady ks. Jerzego Popietuszki 1967-1984, op. cit., p. 106.

${ }^{6}$ Cfr. Ibid, p. 105: "[i lontani tornano alla fede] quando hanno visto che la Chiesa è con loro specialmente quando sono soli, quando hanno visto che la Chiesa in nome della fede aiuta tutti: i «suoi» così come i non credenti".

7 J. Popiełuszko, ,Jestem gotowy na wszystko”, op. cit., pp. 114-115. 
di Dio comincia dall'adozione di un atteggiamento patriottico"". La sopracitata osservazione di don Jerzy sembra essere particolarmente importante per quanto riguarda la comprensione di tutta la sua attività, che dalle autorità polacche di allora veniva vista come attivismo politico e non attività religiosa.

Don Jerzy Popiełuszko voleva stare insieme ai fedeli, voleva condividere con loro il suo tempo. In modo straordinario attirava a sé la gente con la sua semplicità, il suo silenzio, il suo pudore, ma a volte anche con il suo senso dell'umorismo. Tanti hanno scoperto che quel sacerdote era sempre pronto a stare con loro, ad ascoltarli, a pregare insieme. Così, sempre più persone volevano andare da lui, essere in sua presenza. In questo modo don Jerzy ha scoperto ciò che per lui era fonte di gioia: potersi donare agli altri come sacerdote. Sentiva che quel modo di vivere non era facile. In un'intervista confessò: "A volte sento la stanchezza. Mi manca il tempo per tutti. Per me ne ho pochissimo ma non sento lo scoramento"'.

Nei rapporti con i laici don Jerzy si faceva conoscere, prima di tutto, come quello che sta sempre con loro; secondariamente come quello che era lì come sacerdote e come amico. Con il suo esempio insegnava come impegnarsi e come prendere l'iniziativa. Apprezzava molto la collaborazione con i laici. In un'altra intervista disse: "Mi rendo conto che senza di loro non potrei fare molto. Sono già così coinvolti che faranno tanto anche senza di me. Lo testimonia il fatto che quando ero all'estero i giovani si incontravano senza di me e anche durante le vacanze, sostenendo che: «ci mancavi tanto»"

\section{La missione del sacerdote è predicare la parola di Dio}

Don Jerzy Popiełuszko divenne celebre in quasi tutta la Polonia grazie alle sue omelie pronunciate nelle Sante Messe celebrate per la Patria, durante gli anni 1982 - 1984 (cioè fino alla morte). Le parole che rivolgeva ai fedeli lasciano intravedere la sua personalità e la sua spiritualità. Mostrano, quanto don Jerzy apprezzasse la missione sacerdotale di diffondere la parola di Dio e con quanta cautela e rispetto trattasse questo suo impegno. Sapeva che lo ascoltava gente

\footnotetext{
8 Ibid, p. 110.

9 J. Popiełuszko, „Osiadtem” wśród robotników, op. cit., pp. 105-106.

${ }_{10}$ J. Popiełuszko, Stużba bliska kapłaństwu, in: G. Bartoszewski, Zapiski. Listy i wywiady ks. Jerzego Popiełuszki 1967-1984, op. cit., p. 94.
} 
molto diversa perché le sue Messe attiravano non solo tanti operai, ma anche rappresentanti del mondo della cultura e della scienza. Gli appunti che lasciò mostrano quanto scrupolosamente preparava le sue prediche. I suoi amici ricordano che un giorno diede il testo di una sua predica, prima di pronunciarla, a uno degli operai incolti e disse "se la capirà un operaio, la capirà anche un professore". Sapeva che con il Vangelo doveva raggiungere tutti.

Il contesto della vita in Polonia in quei tempi, le persecuzioni costanti ai dissidenti, a "Solidarność" e anche ai credenti, divennero per don Popiełuszko una sfida per insegnare ad amare Dio e il prossimo, parlare della fiducia in Dio e del perdono dei colpevoli, ecc. Spesso gli ascoltatori delle prediche si aspettavano un'altra cosa dall'oratore: una condanna concreta delle persone, un elenco di nomi e cognomi ed anche l'invito ad attività armate contro l'autorità dello Stato. Durante una conversazione privata, quando don Jerzy sentì simili richieste, disse con tristezza: "Io vi parlo così tanto e voi non avete capito niente. Io condanno il male, non l'uomo". Il suo insegnamento, così come tutto il suo atteggiamento venne interpretato dalle autorità comuniste come molto pericoloso. Di conseguenza cercarono di intimidirlo arrestandolo e interrogandolo e pubblicarono contro di lui testi diffamatori sui principali mass-media, perquisirono casa sua, e fecero pressioni sui suoi superiori perché lo facessero tacere. Si accusava don Jerzy di andare oltre i suoi doveri. In risposta a quelle accuse don Popiełuszko disse in una delle sue prediche: "Durante le sante messe in omaggio alla Patria (...) non parlavo mai con mie parole, ma mi lasciavo guidare dal Vangelo e dall'insegnamento del Primate del millennio ${ }^{11}$, Cardinale Stefan Wyszyński e del Santo Padre Giovanni Paolo II"'2.

Quello che diceva don Jerzy fu, dal punto di vista delle autorità comuniste, particolarmente pericoloso. Quelli che lo ascoltavano diventavano sensibili alla verità, al vero bene di ogni singola persona e del paese, alla pace nei cuori e nella società, alla questione della dignità dell'uomo. Però, quello che sembrava ancor più pericoloso per le autorità di allora fu il cambiamento della mentalità e della percezione della realtà in quanti lo ascoltavano. I destinatari delle sue prediche si liberavano della paura paralizzante, che nell'idea dei comunisti avrebbe dovuto penetrare tutta la società per poter imporre così la "verità" del sistema.

11 Titolo onorifico attribuito al card. Stefan Wyszyński.

12 J. Popiełuszko, Kazanie 25 grudnia 1983 r., in: J. Sochoń, Ksiądz Jerzy Popiełuszko. Kazania 1982-1984, Warszawa 2004, p. 127. 
The Person and the Challenges
Volume 3 (2013) Number 2, p. 157-171

Coloro che perseguitavano la "verità" non conoscevano però il mistero cristiano: la Parola di Dio, anche se contrastata, porta sempre frutto. Più don Jerzy ed il suo insegnamento venivano perseguitati, più grandi e buoni erano i frutti tra i fedeli. Le autorità comuniste collaboravano con centinaia di persone allo scopo di spiare don Popiełuszko, intercettare e registrare le sue azioni e per una costante sorveglianza delle persone a lui legate. Questo fatto testimonia significativamente quanto grande era il pericolo che i comunisti vedevano nel suo insegnamento e in tutto il suo servizio sacerdotale. Insieme all'aumento degli sforzi degli agenti dei comunisti, le parole di don Jerzy sull'amore e sul reprimere la voglia di vendetta diventavano sempre più forti e conosciute, raggiungevano persone sempre nuove, attirando alle sue Sante Messe migliaia di persone. L'insegnamento di don Popiełuszko divenne una scintilla per la trasformazione di tantissime persone in tutta la Polonia. Portò molti in Chiesa, li avvicinò ai sacramenti, insegnò come vivere nella verità, cosa significava la libertà e come viverla. Il suo insegnamento portò tanti sulla strada del servizio assiduo nei confronti del prossimo. Il suo esempio mostra come, nella vita di ogni sacerdote, la missione principale sia la predicazione della Parola di Dio.

\section{La missione del sacerdote è impartire i sacramenti}

Sulla base dei testi di don Popiełuszko ed anche analizzando il suo lavoro sacerdotale si può sostenere che la celebrazione dei sacramenti costituiva il centro delle sue attività quotidiane. Il suo ministero quotidiano con i parrocchiani, gli operai nei loro posti di lavoro, tra gli studenti, i lavoratori della Sanità, aveva come scopo portare le persone ai sacramenti: "Lo scopo fondamentale è che le sofferenze che le persone vivono ogni giorno al lavoro, nelle prigioni, per strada, non vadano perse. Questo è il ruolo del prete: indirizzare le sofferenze del Popolo a Dio tramite il sacrificio della Santa Messa, di modo che Dio possa trasformarle in Grazia, necessaria per rafforzare la speranza della gente, per perseverare nelle opere di bene, per lo sviluppo della fraternità e la solidarietà tra la gente"13.

Nella memoria di tanti suoi contemporanei don Popiełuszko rimase come il sacerdote che, non solo era sempre pronto a stare tra la gente e ad ascoltarla, ma pronto anche a confessare e a celebrare la Santa Messa. Se per esempio stava

\footnotetext{
${ }^{13}$ J. Popiełuszko, ,,Jestem z tymi ludźmi w każdej sytuacji”, op. cit., p. 117.
} 
con gli operai scioperanti nello stabilimento metallurgico, o con gli studenti che scioperavano all'Accademia Medica o nella Scuola per Ufficiali dei Vigili del Fuoco a Varsavia, andava sempre pronto a celebrare la Santa Messa. La sua presenza spesso era il primo passo di Cristo che andava verso di loro. Grazie al suo sevizio anche tanti adulti si accostavano al sacramento del battesimo. Tanti invece, dopo anni, di nuovo si confessavano e facevano la comunione. Quando, dopo esser stato tra gli studenti dell'Accademia medica, gli fu chiesto quali erano le sue impressioni, sostenne che: "I giovani lottavano per i loro diritti. Alla facoltà di medicina, ero lì - su richiesta dei giovani - di giorno e di notte. Ogni giorno celebravo la Santa Messa e confessavo. Si può dire che avevano luogo autentiche conversioni. Ricordo uno studente che piangendo in un angolo, mi ha chiesto di prepararlo al battesimo. Un altro studente mi ha rivelato che non si confessava dai tempi della Prima Comunione" ${ }^{\prime 14}$.

Don Jerzy pensava che la celebrazione dei sacramenti nell'ambiente degli operai fosse un compito primario di ogni sacerdote e la considerava come una collaborazione con Cristo nella Sua missione nel mondo. Quello che faceva sempre più spesso si legava ai Sacramenti, specialmente all'Eucaristia. Sapeva che grazie a ciò lo Spirito di Cristo penetra tutta le attività umane ed anche il suo lavoro quotidiano. Perciò don Jerzy cercava spesso di far vedere agli operai il legame tra l'Eucaristia e il loro lavoro, anche un semplice lavoro fisico. "È poi una cosa normale che l'uomo «scopra», veda Dio anche fuori del tempio - lì dove abita, dove riposa. La Chiesa ha sempre aspirato ad una tale coscienza dei credenti. Per così tanti anni abbiamo insegnato: prega al lavoro ed anche tramite il lavoro, che Cristo sia con te sul posto di lavoro. Cosa chiedeva il Papa [Giovanni Paolo II]?: «Aprite le porte a Cristo». Tutte le porte dei paesi, dei sistemi [politici ed economici] ma anche degli uffici, delle fabbriche. È successo così. Sono stati proprio loro - gli operai - a chiederci le Sante Messe sul posto di lavoro. All'inizio forse ciò era legato al senso di pericolo, alla ricerca di appoggio nei momenti difficili degli scioperi. Però con il passare del tempo aveva assunto un altro significato: che Cristo e la Chiesa costruiscano con loro la quotidianità del lavoro, dello sforzo, del servizio per gli altri"'15.

Don Jerzy ci teneva molto che sempre più persone pregassero e si accostassero ai sacramenti. Sapeva che la preghiera era in grado di vincere le avversità più grandi, anche il disumano sistema comunista. Da quel fatto nasceva la sua

\footnotetext{
${ }^{14}$ J. Popiełuszko, Stużba bliska kapłaństwu, op. cit., p. 94.

15 J. Popiełuszko, „Osiadtem” wśród robotników, op. cit., p. 104.
} 
preoccupazione per la Santa Messa. Proprio della Messa parlò in una delle sue prediche: "La Santa Messa è la forma più perfetta di preghiera che il popolo dei fedeli porta a Dio - Padre dei popoli e delle nazioni. Tramite la nostra preghiera vogliamo servire Dio e la gente. Vogliamo includere Dio nell'ambiente dove ci sono i problemi difficili e dolorosi della nostra Patria"16. È significativo che quando qualcuno voleva incontrarsi con don Jerzy per parlare con lui, lui rispondeva: "Venga in chiesa, alla Messa, e dopo parleremo". Don Popiełuszko appare come l'esempio di sacerdote che riconobbe quello che fu più importante per la sua missione nel mondo. Sapeva che la sua forza non era la sua capacità ma la grazia, la quale, tramite lui stesso, andava da Dio ai fedeli - specialmente nei sacramenti.

\section{La missione del sacerdote è confermare nella speranza}

Una delle caratteristiche più significative del sistema comunista polacco consisteva nel togliere alla gente la speranza nel futuro, la speranza che portava al di là del presente. Tutta la vita dell'uomo, tutti i suoi aspetti (famiglia, lavoro, riposo, convinzioni, opinioni, ecc.) nello stato totalitario sarebbero dovuti essere sottomessi all'unica "verità" che diffondeva il sistema. Quella "verità" limitava l'uomo al suo "qui' e "ora", lo racchiudeva nello spazio delle sue attività, nel mondo materiale che non aveva la possibilità di significare nient'altro. La gente poteva parlare solo della vita terrena creata dall'uomo, la vita terrena che si doveva sottomettere totalmente all'uomo, il che nella realtà significava la subordinazione allo Stato. Ad una simile concezione materialista non poteva aderire quell'uomo che porta la speranza cristiana nel cuore.

Don Popiełuszko vedeva la missione del sacerdote in modo molto chiaro e così anche la sua missione particolare: parlare di Cristo che è l'unica speranza, sia per una singola persona, sia per tutte le società. Nelle sue prediche parlava del rafforzamento della speranza ${ }^{17}$, dell'accrescimento della speranza ${ }^{18}$, della forza

16 J. Popiełuszko, Kazanie 27 marca 1983 r., in: J. Sochoń, Ksiądz Jerzy Popiełuszko. Kazania 1982-1984, op. cit., p. 79.

${ }^{17}$ Cfr. J. Popiełuszko, Kazanie 30 października 1983 r., in: J. Sochoń, Ksiądz Jerzy Popiełuszko. Kazania 1982-1984, op. cit., pp. 115 i 119.

${ }^{18}$ Cfr. J. Popiełuszko, Kazanie 27 listopada 1983 r., in: J. Sochoń, Ksiądz Jerzy Popiełuszko. Kazania 1982-1984, op. cit., p. 126. 
della speranza ${ }^{19}$, della vittoria con la forza della speranza e dell'amore ${ }^{20}$, del guardare con la speranza, anche se tra le lacrime, ad un futuro migliore ${ }^{21}$; e anche di quello che non può essere ucciso: la speranza ${ }^{22}$. In un'intervista sostenne con convinzione che i fedeli alle Sante Messe per la Patria "rafforzavano la loro speranza" ${ }^{23}$. Don Jerzy conosceva bene la realtà della Polonia comunista. Sapeva che la speranza tra i fedeli poteva indebolirsi ${ }^{24}$, che si poteva perderla ${ }^{25}$. Sapeva che il sistema nemico alla Chiesa e al suo insegnamento voleva eliminare la speranza teologica e introdurre invece quella laica, separata da $\mathrm{Dio}^{26}$. In quella situazione don Popiełuszko in modo chiaro indicava Dio come la fonte della vera speranza ${ }^{27}$, la speranza che porta verso un futuro luminoso, soprattutto grazie alla preghiera ${ }^{28}$. Nel rapporto dell'anno 1983 presentato alla Curia Metropolitana di Varsavia don Jerzy scrisse: "Com'è stato il mio lavoro tra gli operai lo sa solo Dio onnipotente. Lo sa anche la gente che ho fatto avvicinare a Dio, ed in cui ho rafforzato la speranza e che ho purificato dall'odio" 29 .

Nella Polonia del dopoguerra la Chiesa fu l'unica alternativa al sistema comunista. La Chiesa fu un'oasi per chi voleva parlare della speranza diversa da quella legata alla linea ufficiale delle idee del partito comunista che determinava dei fini dettati dall'ex U.R.S.S. Don Popiełuszko, insegnando sulla speranza cristiana, indicava Cristo come la fonte di essa. Si dimostrò un prete preoccupato per le cose principali dello sviluppo spirituale dei fedeli e anche

${ }^{19}$ Cfr. J. Popiełuszko, Kazanie 30 października 1983 r., op. cit., p. 115.

${ }^{20}$ Cfr. ibid., p. 115.

${ }^{21}$ Cfr. J. Popiełuszko, Kazanie 27 listopada 1983 r., op. cit., p. 121.

${ }^{22}$ Cfr. J. Popiełuszko, Kazanie 26 sierpnia 1984 r., in: J. Sochoń, Ksiadz Jerzy Popiełuszko. Kazania 1982-1984, op. cit., p. 163.

${ }_{23}$ J. Popiełuszko, ,Jestem z tymi ludźmi w każdej sytuacji”, op. cit., p. 116.

${ }^{24}$ Cfr. J. Popiełuszko, Kazanie 29 maja 1983 r., in: J. Sochoń, Ksiadz Jerzy Popietuszko. Kazania 1982-1984, op. cit., p. 77.

${ }^{25}$ Cfr. J. Popiełuszko, Kazanie 26 lutego 1984 r., in: J. Sochoń, Ksiądz Jerzy Popiełuszko. Kazania 1982-1984, op. cit., p. 143; J. Popiełuszko, Kazanie 29 maja 1983 r., op. cit., p. 90.

${ }^{26}$ Cfr. J. Popiełuszko, Kazanie 25 września 1983 r., in: J. Sochoń, Ksiadz Jerzy Popiełuszko. Kazania 1982-1984, op. cit., p. 107.

${ }^{27}$ Cfr. J. Popiełuszko, Kazanie 28 sierpnia 1983 r., in: J. Sochoń, Ksiądz Jerzy Popiełuszko. Kazania 1982-1984, op. cit., p. 103; J. Popiełuszko, Kazanie 25 grudnia 1983 r., op. cit., p. 131; J. Popiełuszko, Kazanie 29 maja 1983 r., op. cit., p. 92.

${ }^{28}$ Cfr. ibid., p. 89.

${ }^{29}$ W. Miziołek, Przedmowa, in: J. Sochoń, Ksiądz Jerzy Popiełuszko. Kazania 1982-1984, op. cit., p. 11. 
per il loro modo di vivere in uno stato totalitario. Con il suo insegnamento e con il suo atteggiamento mostrava la fiducia nella speranza cristiana, che assumeva un significato particolare nel contesto delle sofferenze e che era, allo stesso tempo, un invito ad agire.

Con il suo insegnamento riguardante la speranza cristiana, don Jerzy si iscrisse nella corrente indicata dalla costituzione Gaudium et spes del Concilio Vaticano II e anche nell'insegnamento di Giovanni Paolo II sulla speranza. Sia questo $\mathrm{Papa}^{30}$, sia don Jerzy possono essere chiamati testimoni della speranza. La teologia della speranza di don Popiełuszko è in armonia con il messaggio del Papa polacco. Per entrambi è incentrata su Dio e sottolinea il significato e la missione della Chiesa nel mondo.

Il coronamento ed anche la conferma definitiva dell'insegnamento di don Jerzy sulla speranza è il suo martirio, il quale, come affermava Giovanni Paolo II nell'Esortazione Apostolica Ecclesia in Europa, risultò "la suprema incarnazione del Vangelo della speranza"31. Le parole del Papa in questo documento sembrano riferirsi a don Jerzy e si può dire che questo sacerdote ha servito il Vangelo della speranza con la sua morte, e insieme agli altri martiri "esprimono in grado sommo l'amore e il servizio all'uomo, in quanto dimostrano che l'obbedienza alla legge evangelica genera una vita morale e una convivenza sociale che onora e promuove la dignità e la libertà di ogni persona" ${ }^{\prime 32}$.

\section{La missione del sacerdote è morire per la fede}

All'inizio del terzo millennio del cristianesimo nella Lettera Apostolica Novo Millennio Ineunte (6 gennaio 2001) Giovanni Paolo II scrisse: "La Chiesa ha trovato sempre, nei suoi martiri, un seme di vita"33. Invece nella precedente Esortazione Apostolica Pastores Dabo Vobis (25 marzo 1992) dedicata alla formazione dei sacerdoti nelle attuali circostanze, sostenne che si nota, nell'ambito propriamente religioso e cristiano: "una splendida testimonianza del martirio da parte delle Chiese del Centro-Est europeo"34. In queste parole

\footnotetext{
${ }^{30} \mathrm{Cfr}$. G. Weigel, Il testimone della speranza, Milano 2005.

${ }^{31}$ Giovanni Paolo II, Esortazione Apostolica Ecclesia in Europa, n. 13.

${ }^{32}$ Ivi.

${ }^{33}$ J. Popiełuszko, Lettera Apostolica Novo Millennio Ineunte, n. 41.

${ }^{34}$ J. Popiełuszko, Esortazione Apostolica Pastores Dabo Vobis, n. 6.
} 
si nota il riferimento al martirio di un sacerdote polacco, che il Papa conosceva benissimo - don Jerzy Popiełuszko. Guardando la sua vita e la sua morte da martire e volendo vedere nella sua persona quello che sembra una regola principale del servizio sacerdotale, si può formulare la tesi che uno dei doveri del sacerdote sia la formazione in se stesso della disposizione a morire per la fede, all'eroico dichiararsi a favore di Gesù Cristo.

Il giorno prima della morte di don Jerzy Popiełuszko, ebbe luogo l'evento seguente. Il Vescovo Zbigniew Kraszewski, ausiliare nell'Arcidiocesi di Varsavia, preoccupato per la sicurezza di questo sacerdote, lo avvisò che avrebbe dovuto essere prudente e che i funzionari dell'autorità statale comunista potevano spingersi a fargli del male. Don Jerzy, però, confessò che aveva già passato il limite della paura e che non aveva più timore di niente e che era pronto a tutto. Nelle prediche ricordò che la morte delle vittime del sistema totalitario, delle vittime unite a Dio, non era solo un evento di carattere politico ma - nelle circostanze presenti allora in Polonia - era anche un evento religioso e poteva essere un dono che l'uomo porta a $\mathrm{Dio}^{35}$. In una predica parlava della morte degli operai assassinati come di un sacrificio: "Affidiamo a Te, Signore, gli operai che difendendo i loro diritti umani che hanno sparso il loro sangue e hanno sacrificato la loro vita. Dona loro, Signore, un premio eterno nel Tuo regno" ${ }^{\prime 36}$. Era convinto che il martirio avrebbe portato buoni frutt ${ }^{37}$. La garanzia, per lui, era Gesù Cristo che aveva vinto la morte e operava nella storia: "La morte di Gesù Cristo sulla croce, a dispetto del piano di quelli che Lo avevano condannato, non è stata una sconfitta ma una vittoria. Il sangue di Cristo sparso sulla croce diventò fonte di salvezza. Aprì all'umanità il ritorno alla casa del Padre nel regno dei cieli, il regno della verità, dell'amore, della giustizia e della pace. Il dovere di costruire un regno basato su questi principi Gesù Cristo lo affidò a tutti quelli che avevano stretto l'alleanza con Lui tramite il sacramento del Battesimo. Il popolo polacco da più di mille anni unito a Cristo e al suo insegnamento fu sempre fedele a Dio, alla Chiesa e alla Patria. Le parole «Dio e Patria» furono un elemento indivisibile della storia del nostro popolo. Il popolo polacco sapeva sempre unire il sacrificio della vita e della sofferenza

${ }^{35}$ Cfr. J. Popiełuszko, Kazanie 27 listopada 1983 r., op. cit., pp. 125-126.

36 J. Popiełuszko, Kazanie 25 kwietnia 1982 r., in: J. Sochoń, Ksiądz Jerzy Popiełuszko. Kazania 1982-1984, op. cit., p. 42.

${ }^{37}$ Cfr. J. Popiełuszko, Kazanie 26 grudnia 1982 r., in: J. Sochoń, Ksiadz Jerzy Popiełuszko. Kazania 1982-1984, op. cit., p. 65; J. Popiełuszko, Kazanie 27 listopada 1983 r., op. cit., p. 123. 
con il sacrificio di Gesù Cristo, perché, grazie a quell'unione, non si perdesse niente, ma anzi diventasse una sostanza nutriente per le future generazioni”" ${ }^{38}$.

\section{Conclusioni}

Il fenomeno di don Jerzy Popiełuszko apparì in tutta la sua intensità dopo la sua morte da martire. Dal momento in cui fu comunicata la sorte di don Jerzy, si iniziò a sostenere che la sua morte fosse stata un martirio per la fede e che lui fosse un martire. Dal 3 novembre 1984, cioè dal giorno del funerale di don Popiełuszko, la sua tomba fu visitata da pellegrini provenienti da tutta la Polonia e da tutte le parti del mondo. La documentazione raccolta nella parrocchia di San Stanislao Kostka a Varsavia, dove si trova la sua tomba, lascia ritenere che finora circa 20 milioni pellegrini, tra cui circa 400 mila stranieri da più di 140 paesi, l'abbiano visitata. Alla tomba di questo beato martire arrivano vescovi e sacerdoti da tutto il mondo. Il 14 giugno 1987 pregò lì anche Giovanni Paolo II e il Cardinale Joseph Ratzinger come Prefetto della Congregazione per la Dottrina della Fede (25 maggio 2002).

Don Jerzy Popiełuszko, beatificato il 6 giugno 2010 durante l'anno in cui tutta la Chiesa vive l'Anno Sacerdotale, appare come un chiaro esempio per i sacerdoti. Ci ricorda che la santità è possibile da realizzare e da raggiungere in tutte le circostanze storiche. Don Jerzy appare anche come un esempio di sacerdote molto impegnato che percorse la strada verso la santità collaborando con tanti laici, i quali si distinguevano per una particolare prontezza alla collaborazione e per la preoccupazione per il bene della Chiesa nel mondo che lotta contro la Chiesa Dio, e i suoi sacerdoti.

\section{Bibliography}

Popiełuszko J., Kazania 1982-1984, Warszawa 2004.

Zapiski. Listy i wywiady ks. Jerzego Popietuszki 1967-1984, G. Bartoszewski (ed.), Warszawa 2009.

Popiełuszko J., Rozważania różańcowe, in: M. Kindziuk, Ksiądz Jerzy zwyciężał dobrem, Warszawa 2009, p. 190-198.

${ }^{38}$ Ibid., p. 121. 
Burgoński P., Polski patriotyzm $w$ dobie komunizmu. Koncepcja patriotyzmu $w$ nauczaniu księdza Jerzego Popietuszki, „Warszawskie Studia Teologiczne” XXIII/1 (2010), p. 241252.

Kaczmarek T., Jan Paweł II wobec świadectwa ks. Jerzego Popiełuszki, „Ateneum Kapłańskie” 143 (2004), p. 495-507.

Kindziuk M., Świadek prawdy. Życie i śmierć ks. Jerzego Popiełuszki, Częstochowa 2004.

Malacki Z., Stuga Boży, ksiadz Jerzy Popietuszko, Warszawa 2002.

Nitecki P., Znak zwycięstwa. Ksiądz Jerzy Popietuszko (1947-1984), Warszawa 1998.

Smuniewski C., Kościół w myśli błogosławionego ks. Jerzego Popiełuszki, „Warszawskie Studia Teologiczne" XXIII/1 (2010), p. 215-230.

Sochoń J., Ksiądz Jerzy Popiełuszko: w kręgu kultury przykościelnej, „Warszawskie Studia Teologiczne" XXIII/1 (1010), p. 231-240. 